\title{
About Possible Role of Negative Pressure Waves in Geophysical Processes
}

\author{
Fuad H. Veliev ${ }^{1} \&$ Ibrahim S. Guliyev ${ }^{2}$ \\ ${ }^{1}$ Baku Higher Oil School, Baku, Azerbaijan \\ ${ }^{2}$ Geology Institute, Azerbaijan National Academy of Sciences, Azerbaijan \\ Correspondence: Fuad H. Veliev, Baku Higher Oil School, AZ1025, 30 Khojaly ave., Baku, Azerbaijan. \\ Tel: 994-503-760-831. E-mail: fuadveliev@hotmail.com
}

\author{
Received: August 6, 2014 Accepted: August 26, 2014 Online Published: August 30, 2014 \\ doi:10.5539/apr.v6n5p78 URL: http://dx.doi.org/10.5539/apr.v6n5p78
}

\begin{abstract}
There are different model views on the mechanism of extreme geologic processes. Nevertheless, many principal questions remain unanswered because the models don't characterize sufficiently the peculiarities of these processes. Here is proposed new approach to mechanism of evolution and appearance of extreme geologic processes on the basis of Negative Pressure phenomenon.
\end{abstract}

Keywords: negative pressure, wave, hydraulic system, subsurface environment, volcanic eruptions, earthquakes

\section{Introduction}

Identification of particular features and driving mechanism of rapidly going geologic phenomena such as earthquakes, eruptions of magmatic and mud volcanoes, geyser activity etc still remain one of the key challenges of geoscience nowadays. The extreme geologic events are considered as awakening of dormant subsurface environment releasing huge amount of energy and disintegrated masses (magmas, breccias coal and ore dust, gases etc) which occurrence rate is unmatched with geologic processes duration lasting for millions of years. The results of durable theoretical and experimental investigations indicate that the Negative Pressure apparently is one of the driving factors in mechanisms of evolution and manifestation of geophysical catastrophes as volcanic eruptions and earthquakes.

The Negative Pressure is known to be one of the metastable states in which liquids can be extended up to a certain limit without breaking of their continuity (Hayward, 1971). Negative pressure as a scientific notion has actually been known for quite a long period of time in the realm of thermodynamics, although, the phenomenon itself and its impact on natural processes have not been studied and understood completely so far.

Negative Pressure occurs in many natural and technological processes. It has been recognized that arboreal liquid moves in ascending order inside a tree trunk ought to negative pressure. It is argued that negative pressure is very crucial in some vital functional systems of living organisms, particularly in blood circulation system.

Theoretical assessment demonstrate feasibility to arrange stretching forces in pure liquids (without impurity, bubbles etc) relevant to huge negative pressure (10 MPa and more).

\section{Experimental}

Values of Negative Pressure obtained experimentally are certainly lower, and in all numerous lab investigations beginning from pioneering one carried out as long ago as 1843 by F. M. Donny, who has achieved just $-0.11 \mathrm{MPa}$ to tests carried out by Briggs (1950) where a record value of negative pressure $(-42.5 \mathrm{MPa})$ has been reached, the effect was mainly conditioned by degree of the liquid-vessel purity(Hayward, 1971).

Long-term investigations carried out comparatively not long ago to reach negative pressure in real impure heterogeneous liquids were crowned with success, and direct wave of negative pressure in natural hydraulic systems as tap water, crude oil, solutions etc in pipe and porous medium was induced experimentally (Veliev, 1983). A procedure to achieve the negative pressure was based on the idea of abrupt application of stretching efforts under which steam generation centers in liquid-vessel system as bubbles, germs, impurities have not time to manifest themselves. In such circumstances liquid purity is not a dominant factor, and there might emerge a phenomenon with overheated liquid and negative pressure. 
The results of numerous tests show that if the liquid system in a pipe or porous medium being under initial excess pressure is unclosed quickly enough (the process' characteristic time is much less than the time of relaxation), then a pressure falling sharply may become negative for a short time in certain conditions. It was determined that the Negative Pressure wave generated in the system resembles overturned soliton wave with one but negative hump and propagates with a sound velocity. Although the appearance of the negative pressure is almost instantaneous it leads to significant overheating, spontaneous evaporation and gas emission in the system, associated with essential temperature falls and cavitation process. It was revealed that a passing of Negative Pressure waves through system results in considerable reduction of hydraulic resistance, increasing of permeability of porous medium, and consequently, intensification of filtration process.

Figure1 represents the typical variation of pressure with time in crude oil stream in two test points along the pipe under initial values of pressure and temperature $\mathrm{P}_{0}=0.7 \mathrm{MPa}, \mathrm{T}_{0}=298 \mathrm{~K}$.

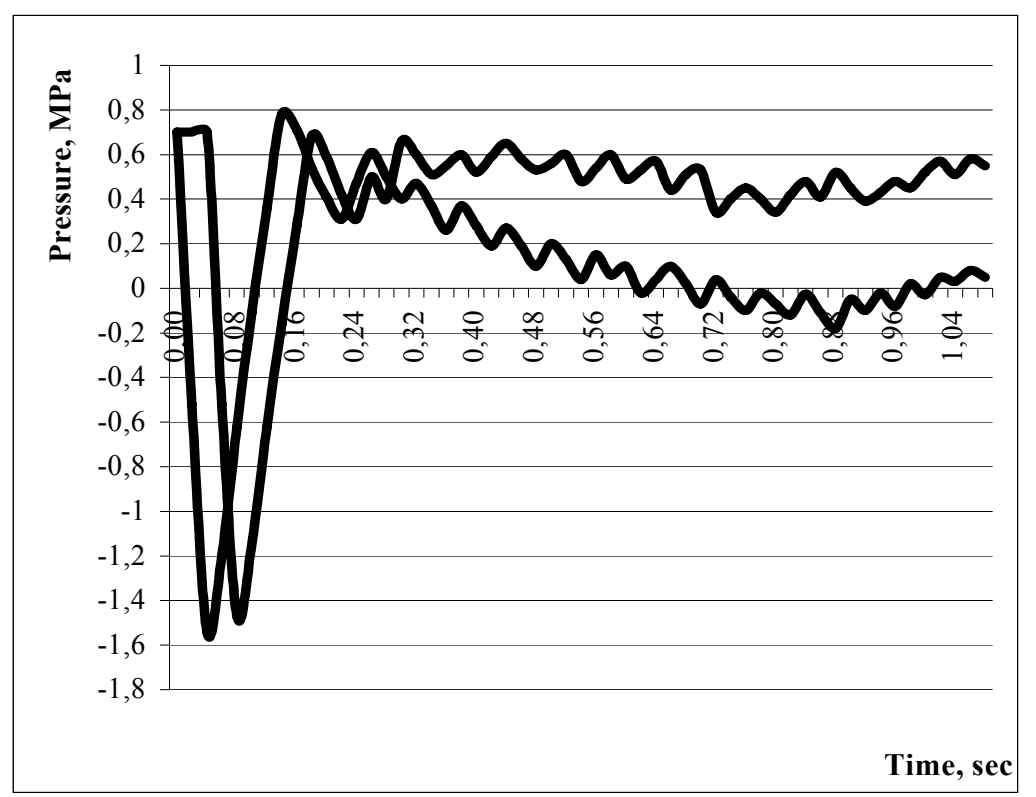

Figure 1. Variation of pressure in two test points of an oil stream in a pipe

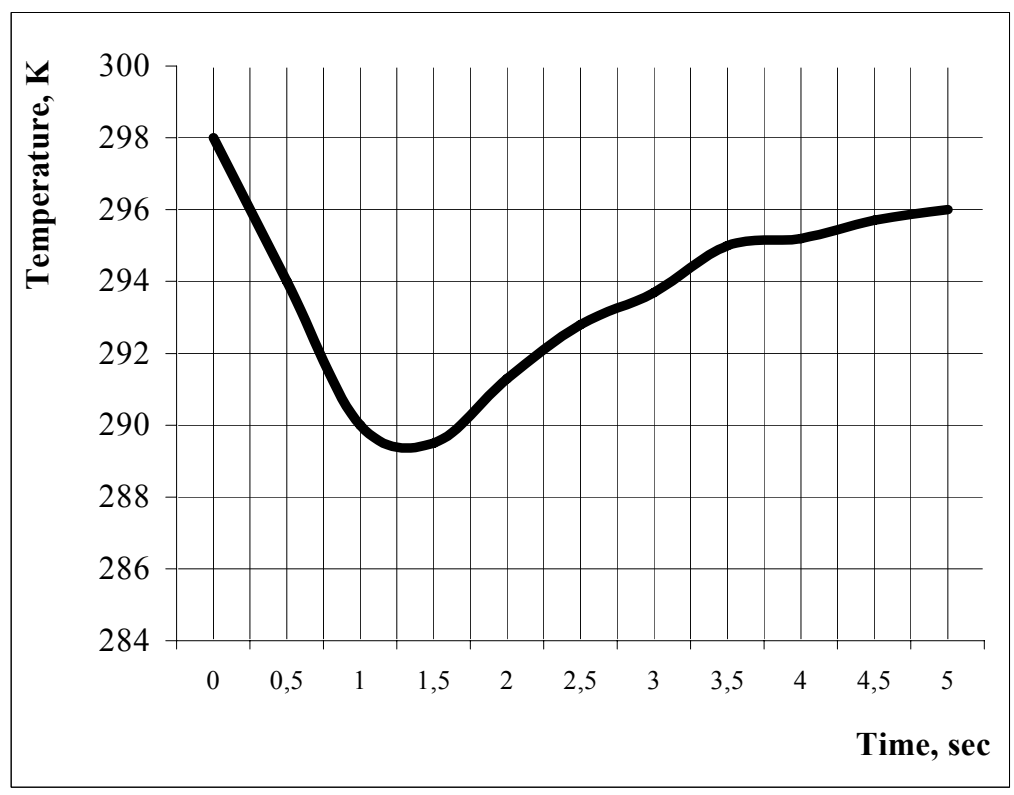

Figure 2. Variation of a stream temperature 
As one can see the generated negative pressure appears for a short time, reaching the maximum value of $-1.5 \mathrm{MPa}$ during of $0.04 \mathrm{sec}$. It was determined that wave of Negative Pressure resembling overturned soliton wave with one but negative peak propagates with speed of sound. The process is followed by quick considerable fall in the temperature of the stream $\left(\sim 7^{\circ}-10^{\circ}\right)$ after which it is slowly restored apparently due to heat transfer process accompanied by processes of cavitation, reverse condensation of steam, dissolving of the extracted gas (Figure 2).

It should be underlined that forming of negative pressure waves may be result of transformation of ordinary regular waves rebounding from various obstacles, free surfaces, interphase boundaries (Couzens \& Trevena, 1969). A wide range of theoretical and pilot modeling works to study the impact of negative pressure on some physical characteristics of natural systems has been carried out on this basis (NATO Science Series, 2001).

The manifestation of Negative Pressure phenomenon was repeatedly observed during of oil-well drillings and operations as a result of technological actions associated by sharp displacement of different equipments (drilling tools, round-trip pipes etc) within the well and abrupt disclosing of a hole mouth. The negative pressure may cause very hard complications as dreadful open showings of oil and gas, column crushing, borehole wall collapse, gryphon appearance (Veliev, 2002).

\section{Discussion}

Apparently main realm where negative pressure wave might appear is the subsurface environment that represents anisotropic environment with extremely wide range of physical, chemical and structural characteristics, huge masses and tremendous volumes, powerful inducing factors. Results of investigations mentioned above indicate on inevitability of generation of the negative pressure waves in the Earth interior. Factors initiating the appearance of these waves in the subsurface medium may be tectonic displacement and faults, abrupt dislocation of rocks and fluids. Negative pressure waves may also appear as a result of transformation of regular seismic waves rebounding from various underground obstacles, surfaces and barriers.

Negative pressure wave can provoke "excitation" of subsurface environment in a focus quite remotely located from the source point of the negative pressure wave. It is noteworthy, that some non-linear anisotropic structures in subsurface durable to overcome huge compression forces might be destroyed by very small stretching forces ought to negative pressure.

Rock pore space charged with gases and formation water under high pressure and temperature, gas hydrates exemplify extremely unstable systems in cases with abrupt temperature and pressure alteration. The negative pressure wave appeared in any space point entails spontaneous boiling and vaporization in the hydraulic system, intensive gas release, cavitation process, increasing permeability, reduction of hydraulic resistance, pseudo-liquefaction, that is, all that foregoes and accompanies extreme geologic phenomena as eruptions of mud and magmatic volcanoes, earthquakes in the lithosphere, geyser activity etc.

Mud volcanism, according to the up-to-date ideas is driven by rapid basin subsidence extensive gas generation and huge amount of water released while catagenesis (Ivanov \& Guliyev, 1989). With high sedimentation rate and sediment compaction as well as pore water saturation dominantly with hydrocarbon gases, there appear unbalanced water-hydrocarbon-liquid and solid (gashydrates) systems under tremendous pressure close or exceeding geostatic one. At such circumstances there appear a staggered faulting system and abrupt pressure drop. There emerges a distinctive paragenesis of overpressured and underpressured zones when abnormal high pressure causes abnormally low pressure including the negative one. Subsequent propagation of negative pressure wave entails instantaneous water boiling, spontaneous degassing of formation water charged with hydrocarbons lead to intensive dynamic effects, gas migration, cavitation effects, sediments under-compaction right up to pseudo-liquefied state. As a result of buoyancy effect of under-compacted deposit, squeezing out to the surface and gas releasing through the open conduits, their subsequent inflammation - lead to mud volcano eruption.

Apparently, Negative Pressure takes a significant part in magmatic volcano eruption. Water-to-vapor transfer with thousand fold volumetric increment in the focus of eruption is one of the key factors in magmatic volcano and geyser occurrence. Traditionally this process is assumed to be associated with high temperature impact. It is not excluded that Negative pressure occurrence is capable to catalyze and intensify those processes, particularly long-term vibrations called volcanic tremor might be a consequence of emerging pass-along negative pressure wave.

The other possible consequence of Negative Pressure occurrence might be earthquakes in lithosphere. Their spatial distribution does not correlate with most accepted hypothesis of abrupt rupture along seismicity generating faults because sedimentary rocks particularly the young one and saturated by fluids has no necessary strength for abrupt rupture. None the less in younger basins, for example, in South Caspian basin significant amount of earthquakes 
occurs in sedimentary body. One of the probable reasons for their appearance might be negative pressure waves in unbalanced environment, for example, gas-charged pore-water, gas-hydrates and other metastable systems.

The Negative Pressure phenomenon, evidently, takes a significant part in subsurface processes and inevitability of generation of negative pressure waves in the Earth interior must be taken into account in evaluating of geophysical processes. Extreme dynamic processes in the underground medium actually can be considered as a synergetic manifestation of the Negative Pressure together with other thermohydrodynamical factors.

Nowadays, however, there is a need for special comprehensive experiments to understand entirely the scale and the role of the negative pressure in conventional geologic phenomena. Required investigations can be realized completely through adoption and implementation of international scientific program for field registration and monitoring of Negative Pressure waves in different regions, especially, in zones of high seismic activity.

Revelation and control of Negative Pressure waves in the Earth's entrails would be a result of a paramount importance for science and practice. It would completely change views on mechanisms of evolution and appearance of different geodynamic processes, give as a matter of fact an additional chance for control of geologic medium, elaborate principally new approach in evaluating of geophysical processes, create highly effective energy saving technologies.

\section{References}

Couzens, D. C. F., \& Trevena, D. H. (1969). Critical Tension in a Liquid under Dynamic Conditions. Nature, 222 , 437. http://dx.doi.org/10.1038/222473a0

Hayward, A. T. J. (1971). Negative Pressure in Liquids: Can it be harnessed serve man? American Scientist, $59,434$. Imre, A. R., Maris, H. J., \& Williams, P. R. (Eds.) (2001). NATO Science Series, Liquids Under Negative Pressure.

Ivanov, V. V., \& Guliyev, I. S. (1989). Physical-chemical Model of Mud Volcanism, 1989. In Problems of oil-gas potential in the Caucasus. Moscow: Nauka.

Veliev F. H. (2002). Energy Saving Technologies on the Basis of Negative Pressure Phenomenon, 2002. Proceedings of International Congress “Energy, Ecology, Economy”. Baku.

Veliev, F. H. (1983). About the Negative Pressure. Injenerno-Fizicheskiy Jurnal (Journal of Engineering Physics), $44,2$.

\section{Copyrights}

Copyright for this article is retained by the author(s), with first publication rights granted to the journal.

This is an open-access article distributed under the terms and conditions of the Creative Commons Attribution license (http://creativecommons.org/licenses/by/3.0/). 